environmental services workers who had passed the temperature and symptom screening evaluation on entry to the campus. Samples were obtained from both nares of consenting individuals and ran daily on a validated polymerase chain reaction (PCR) platform.

Results: A total of 1394 employees consented to participate by June 15, 2020 and none of them had a positive result for COVID-19. Thus, the prevalence rate among asymptomatic employees was zero during this period (95\%CI $0 \%-0.26 \%$ ). Thirteen employees developed symptoms after initial enrollment and testing of which 1 tested positive for COVID-19. Based on these data, we estimate a monthly incidence rate of 0.8 new COVID-19 cases per 1000 asymptomatic employees (95\%CI $0.2-4.4$ per 1000 ). Of note, at the time of submission the $\mathrm{R}_{0}$ for our region was 1.4 , and the prevalence of COVID-19 infection among symptomatic employees was 9.9\% (68/686 tested in our system, $95 \% \mathrm{CI}$ : 7.8\%-12.3\%).

Conclusion: We did not identify any COVID-19-positive asymptomatic hospital employees who passed screening measures. This suggests very low risk of nosocomia transmission to other employees, patients, and families. Along with low community prevalence and capture of COVID-19-positive symptomatic employees, we could confidently advise staff that universal surgical masking and eye protection were likely adequate to prevent significant exposure.

Disclosures: All Authors: No reported disclosures

464. Rapid whole genome sequence typing reveals multiple waves of SARS-CoV-2 spread

Ahmed M. Moustafa, $\mathrm{PhD}^{1}$; Paul J. Planet, $\mathrm{MD}, \mathrm{PhD}^{2}$; Paul J. Planet, $\mathrm{MD}, \mathrm{PhD}^{2}$;

${ }^{1}$ Sackler Institute for Comparative Genomics, American Museum of Natural

History, New York, NY; The Children's Hospital of Philadelphia, Philadelphia,

PA., Philadelphia, PA; ${ }^{2}$ Children's Hospital of Philadelphia/UPenn, Philadelphia,

Pennsylvania

Session: P-14. COVID-19 Epidemiology and Screening

Background: As the pandemic SARS-CoV-2 virus has spread globally its genome has diversified and distinct clones can now be recognized, tracked, and traced. Identifying clonal groups allows for assessment of geographic spread, transmission events, and identification of more virulent or transmissible emerging strains.

Methods: All SARS-CoV-2 genomes $(\mathrm{n}=17,504)$ that are complete and high coverage were downloaded from GISAID on May $17^{\text {th }} 2020$. We developed a GNUbased Virus IDentification (GNUVID) tool that implements a whole genome multilocus sequence typing (wgMLST) scheme composed of all ten ORFs in the SARS-CoV-2 genome. The 10,422 genomes that passed our quality check were fed to the GNUVID tool, which assigned a ST profile to each genome. Global optimum eBURST was then used to cluster the STs in clonal complexes (CCs).

Results: Our ST/CC analysis uncovered strong associations of ST/CCs with certain geographical regions but also dynamic local changes in ST/CC prevalence. We also identified several unexpected putative global transmission events (e.g., from the US to the Middle East and reintroduction to China later in the pandemic). We have made our tool (GNUVID) available so that new WG sequences can be rapidly assigned to an ST/ CC (https://github.com/ahmedmagds/GNUVID).

Conclusion: Our sequence typing system uncovered previously unappreciated transmission events and waves of expansion and replacement of SARS-CoV-2 STs and CCs in different geographical locations, suggesting complex dynamics in viral populations that previously seemed monomorphic. Because, our tool can be rapidly updated with new sequencing data it can track emerging clones and identifying new hotspots.

Disclosures: All Authors: No reported disclosures

\section{Risk Factors Associated with Critical COVID-19 Requiring Mechanical} Ventilation

Robert B. Chris, MD MSc ${ }^{1}$; Rabeeya Khalid, $\mathrm{MD}^{1}$; Urmila Ravichandran, $\mathrm{MS}^{2}$; Mary Ellen Acree, MD; Jennifer Grant, $\mathrm{MD}^{2}$; Chethra Muthiah, $\mathrm{MD}^{2}$; Nirav Shah, $\mathrm{MD}, \mathrm{MPH}^{2} ;{ }^{1}$ University of Chicago (NorthShore), Evanston, Illinois; ${ }^{2}$ NorthShore University HealthSystem, Evanston, Illinois

Session: P-14. COVID-19 Epidemiology and Screening

Background: Mechanical ventilation of patients with COVID-19 is associated with high mortality. Understanding risk factors for developing mechanical ventilation may allow for more targeted monitoring and therapeutics that may improve outcomes.

Methods: We performed a retrospective case series of all patients admitted within thirty days of a positive Sars CoV-2 test to an integrated health system near Chicago, Illinois between March 12 and May 31, 2020. Covariates evaluated included demographics, symptoms on admission, vital signs, medications, comorbidities, census tract data and social history. Univariable analysis was performed and variables with an alpha of 0.05 or less were included in multivariable regression modeling to identify factors associated with mechanical ventilation. The area under the curve (AUC) was used to assess performance of the model.

Results: Of 990 patients admitted with COVID-19 12.6\% (125) were mechanically ventilated. The median age was 68 (interquartile range $55-82$ ), 48.4\% (479) were female and $49.6 \%$ (491) were Caucasian. Independent factors associated with mechanical ventilation included female sex (Adjusted OR [AOR] 0.621, Confidence Interval [CI] 0.427-0.903; $\mathrm{p}=0.0363$ ), body mass index (BMI) (AOR 1.035, CI $1.011-1.060 ; \mathrm{p}=0.0175)$, percent of english speaking population within patient's census tract (AOR 0.989, CI 0.979-0.998; $\mathrm{p}=0.0454)$, respiratory rate (AOR 1.054, CI $1.027-1.083 ; \mathrm{p}=0.0011)$, oxygen saturation $($ AOR 0.922 , CI $0.901-0.943 ; \mathrm{p}<0.0001)$, cerebrovascular accident (CVA) (AOR 0.176, CI 0.051-0.605; $\mathrm{p}=0.0207$ ) and hematologic malignancy (AOR 3.668, CI 1.403-9.590; $\mathrm{p}=0.0261$ ). AUC of the model was $0.8(0.75-0.84)$

Characteristics of patients admitted with COVID-19
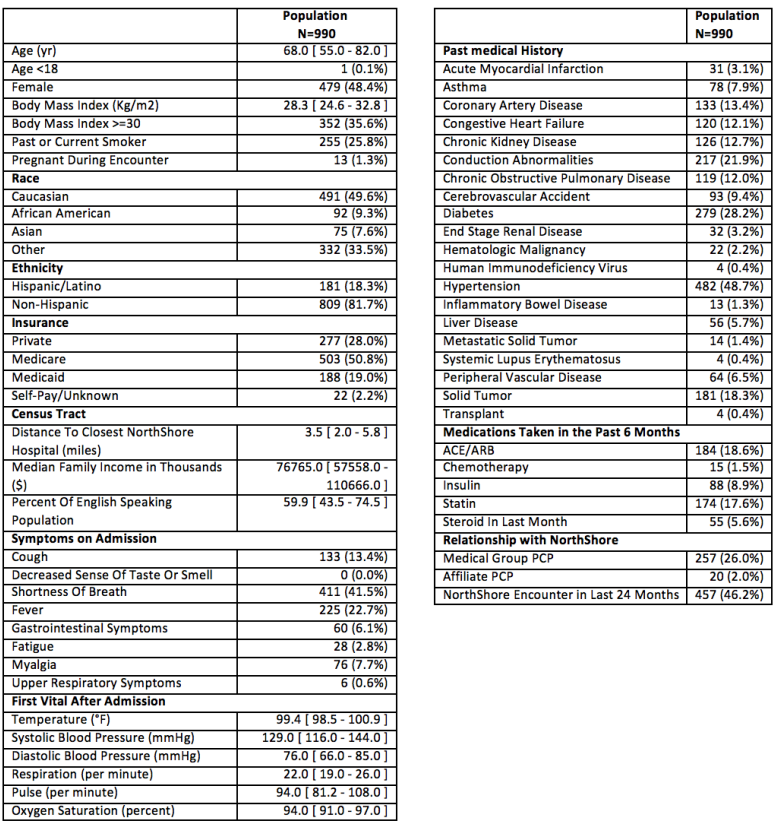

Multivariable logistic regression to identify risk factors associated with mechanical ventilation

\begin{tabular}{|l|r|r|}
\hline & Adjusted Odds Ratio & P-Value \\
\hline Age (yr) & $0.997[0.985-1.009]$ & 0.7023 \\
\hline Female & $0.621[0.427-0.903]$ & 0.0363 \\
\hline Body Mass Index (Kg/m2) & $1.035[1.011-1.060]$ & 0.0175 \\
\hline Percent Of English Speaking Population & $0.989[0.979-0.998]$ & 0.0454 \\
\hline Shortness Of Breath & $1.244[0.844-1.835]$ & 0.3549 \\
\hline Respiration (per minute) & $1.054[1.027-1.083]$ & 0.0011 \\
\hline Pulse (per minute) & $1.005[0.995-1.014]$ & 0.4005 \\
\hline Oxygen Saturation (percent) & $0.922[0.901-0.943]$ & 0.0000 \\
\hline Conduction Abnormalities & $0.634[0.374-1.077]$ & 0.1569 \\
\hline Cerebrovascular Accident & $0.176[0.051-0.605]$ & 0.0207 \\
\hline Hematologic Malignancy & $3.668[1.403-9.590]$ & 0.0261 \\
\hline Non-Hispanic & $1.125[0.706-1.795]$ & 0.6771 \\
\hline
\end{tabular}

Conclusion: $\quad$ Risk factors associated with mechanical ventilation included male gender, elevated BMI, census tract with lower percentage of english speakers, increased respiratory rate, low oxygen saturation, hematologic malignancy and not having a CVA. We suspect that history of CVA may have been associated with overall patien debility in which aggressive measures such as intubation were not deemed appropriate. Identifying patients with risk factors associated with mechanical ventilation may allow for early and targeted interventions to improve outcomes.

Disclosures: All Authors: No reported disclosures

\section{SARS-CoV-2 Diagnosis And Point Prevalence in a Non-Cohorted Tertiary} Care Center

Spencer Schrank, DO ${ }^{1}$; Katherine McAleese, $\mathrm{MD}^{1}$; Amanda B. Spence, $\mathrm{MD}^{2}$, Madhuri Natarajan, $\mathrm{MS}^{3}$; Joseph Timpone, $\mathrm{MD}^{4}$; Joseph Chang, $\mathrm{MD}^{5}$; Gayle p. Balba, $\mathrm{MD}^{6}$; Princy Kumar, $\mathrm{MD}^{6} ;{ }^{1}$ MedStar Georgetown University Hospital, Washington, District of Columbia; ${ }^{2}$ Georgetown University Medical Center, Washington,

District of Columbia; ${ }^{3}$ Medstar Georgetown University Hospital, Washington, DC; ${ }^{4}$ Georgetown University, Washington, DC; ${ }^{5}$ Fellow, Washingon, District of Columbia; ${ }^{6}$ Georgetown University School of Medicine, Washington, District of Columbia

Session: P-14. COVID-19 Epidemiology and Screening

Background: The CDC recommends testing for SARS-CoV-2 in patients who present with symptoms consistent with COVID-19 and to cohort hospitalized patients diagnosed with COVID-19. Up to $35 \%$ of persons infected with SARS-CoV-2 are asymptomatic; however, no recommendations exist for universal testing in hospitalized patients. We assessed the point prevalence of SARS-CoV-2 infection amongst hospitalized patients at a tertiary care center during a time when there was a regional surge of cases.

Methods: Nasopharyngeal SARS-CoV-2 PCR testing was performed on inpatients at Georgetown University Hospital on 4/27/20, excluding those who were SARSCoV-2 positive, tested within 72 hours or admitted to pediatric, psychiatric, labor \& delivery or ICUs. Patients within the hospital were not cohorted based on COVID-19 